\title{
Féeries
}

Études sur le conte merveilleux, XVII $-\mathrm{XIX}{ }^{\mathrm{e}}$ siècle

\section{Un couple fantomatique : le conte et la fable chez quelques fabulistes des Lumières}

A Ghostly Couple: Tale and Fable in the Works of a few Enlightenment Writers

Jean-Noël Pascal

\section{CpenEdition}

1 Journals

Édition électronique

URL : http://journals.openedition.org/feeries/762

ISSN : 1957-7753

Éditeur

UGA Éditions/Université Grenoble Alpes

Édition imprimée

Date de publication : 31 octobre 2010

Pagination : 137-145

ISBN : 978-2-84310-182-3

ISSN : 1766-2842

\section{Référence électronique}

Jean-Noël Pascal, « Un couple fantomatique : le conte et la fable chez quelques fabulistes des

Lumières », Féeries [En ligne], 7 | 2010, mis en ligne le 31 juillet 2011, consulté le 07 septembre 2020.

URL : http://journals.openedition.org/feeries/762

\section{(c) Féeries}




\author{
Jean-Noël Pascal \\ Université de Toulouse-Le Mirail
}

\title{
UN COUPLE FANTOMATIQUE : LE CONTE ET LA FABLE CHEZ QUELQUES FABULISTES DES LUMIÈRES
}

UTANT LE DIRE d'entrée : pour séduisant qu'il puisse nous paraître, le couple conte / fable, vu du moins du côté des fabulistes en vers du temps des Lumières, entre La Motte (I7I9) et Florian (I792), n'est guère problématique. Conte, assez rarement employé dans les textes et à peu près absent dans les paratextes ${ }^{\mathrm{I}}$, désigne en général - et la plupart du temps implicitement — ce qu'on pourrait appeler narration ou récit - ou encore parfois image, d'une manière qui renvoie à l'allégorie — - et l'on se trouve renvoyé à telle formule fameuse dans laquelle le fablier signalait que la leçon devait être insinuée à partir d'une historiette racontée. L'important appareil théorique déployé ${ }^{2}$, essentiellement dans la première moitié du siècle, par des auteurs soucieux, en réduisant la pratique de La Fontaine à des règles applicables par d'autres que lui ou en opérant à son égard un prudent contournement en forme, le plus souvent, de retour à la tradition didactique et rhétorique de la fable, s'il les conduit généralement à s'interroger sur les qualités et le style du récit, ne se préoccupe guère de distinctions terminologiques en dehors du champ générique restreint où il travaille.

I. Dans son Discours sur la fable en tête de ses Fables nouvelles (Dupuis, I7I9, éd. ordinaire in-I2 sans les somptueuses illustrations de Gillot), La Motte évoque bien (p. LIII) les Contes de La Fontaine, mais c'est seulement pour noter qu'il s'y est "exercé longtemps à la narration ". Florian, quant à lui, fait dire au vieillard qui raisonne sur le genre dans son liminaire intitulé De la fable (Paris, de l'imprimerie de P. Didot l'aîné, I792, éd. in-I2) que la fable doit être «une espèce de petit drame» (p. 19), que le fabuliste doit posséder au plus haut point l'art de peindre et «celui de conter gaiement» (p. 20). Et c'est à peu près tout.

2. Sur ces questions, voir principalement J.-N. Pascal, Les Successeurs de La Fontaine au siècle des Lumières, New York et Berne, Peter Lang, 1995 ( $\mathrm{I}^{\mathrm{re}}$ partie, chap. II); «Les successeurs de La Fontaine et la poétique de la fable», dans M.-M. Fragonard, J.-C. Brunon, M. Bideaux et J.-N. Pascal (dir.), Fables et fabulistes, Mont-de-Marsan, éd. InterUniversitaires, I992; "Brèves remarques sur quelques fabulistes des Lumières : une nouvelle idée du genre au service d'idées nouvelles?», dans G. Artigas-Menant et A. Couprie (dir.), L'Idée et ses fables, le rôle du genre, Paris, Champion, 2008. 
En réalité, tout se passe comme si le conte - type narratif pur - fonctionnait en parallèle avec la fable - autre type narratif, mais impur, en raison de sa finalisation morale - sans jamais vraiment la rencontrer, à moins qu'il ne se confonde parfaitement avec elle, jusqu'à en disparaître totalement. Et ce qui en témoigne le plus évidemment - et le plus paradoxalement - , ce sont les titres dans lesquels les deux termes se trouvent réunis, qui sont relativement nombreux dans la période 1750-1780: Fables et contes (1754) de Claude-François Boulenger de Rivery (I725-I758), Fables allemandes et contes français en vers (I770) d'Alexandre-Jacques Du Coudray (I744-I790), Fables et contes philosophiques (I77I) du père Philippe Barbe (1723-1792), Fables, contes et épîtres (1773) de l'abbé Guillaume-Antoine Le Monnier (I72I-I797), etc. La liste n'est pas limitative.

Chez Du Coudray3, la question est vite réglée : les quatre livres de fables, annoncés comme "première partie» et précédés d'un Essai sur le genre en forme de lexique des auteurs antérieurs, sont suivis, en pagination indépendante, d'un "recueil dans le recueil» intitulé Mes trentesix contes, qui est donc la «seconde partie» de l'ouvrage. À la lecture, la distinction générique, même s'il est aisé de repérer les sources des fables (Gellert, Lessing, Lokman) que le poète ne cherche aucunement à celer et de constater que les contes, plutôt médiocres et généralement brefs, appartiennent à la filiation de Vergier plus encore qu'à celle de La Fontaine, ne saute pas aux yeux : la mise en relief de la moralité dans les textes de la première partie n'est pas particulièrement soignée et bien des narrations s'achèvent sur une procédure qu'on retrouve dans ceux de la seconde, la prise à partie des lecteurs.

Dans le recueil de Rivery ${ }^{4}$, qui a précédé Du Coudray dans l'exploitation du fonds allemand s' (Gellert, dont il alterne les adaptations avec d'autres de fables anglaises de Gay et de fables de Phèdre), les choses sont beaucoup moins claires : seul le long texte conclusif (Inkle et Yarico, III, I7) est explicitement désigné comme un conte, alors qu'il est essentiel-

3. Fables allemandes et contes français en vers, avec un Essai sur la Fable, par M. D***, ci-devant mousquetaire, Paris, F. H. Monory, I772, LVI-II6-IO8 pages (et 8 folios de table, non paginés). L'auteur, dans son liminaire, fait état d'une édition antérieure (I770), que je n’ai pas eue sous les yeux.

4. Fables et contes, Paris, Duchesne, I754, LXviII-I29 pages (et ir folios de table, non paginés).

5. Voir J.-N. Pascal, «La découverte des fabulistes européens par les fabulistes français entre 1750 et $\mathrm{I} 820$ ", dans M. Bertaud (dir.), La Littérature française au croisement des cultures, $\mathrm{n}^{\circ} \mathrm{XXII}$ des Travaux de littérature, 2009. 
lement emprunté à une des fables de Gellert ${ }^{6}$, croisée avec un récit du Spectator. Pour le reste, l'Avis de l'éditeur, en fin de volume, ignore la distinction, dont il suppose probablement qu'elle peut être opérée spontanément par le lecteur, et la table des matières, qui renvoie scrupuleusement aux ouvrages utilisés par le poète, ne nous est d'aucune utilité : quand par hasard l'indication du titre abrégé des Fabeln de Gellert est absente et que l'on pourrait supposer que l'original transposé ressortit à la typologie du conte, l'on est vite détrompé, comme par exemple dans le cas de L'Avengle et le Boiteux (Rivery, III, 7, p. 95-96), pièce que Florian a rendu assez fameuse ${ }^{7}$ et qui est bien une fable (ou une parabole), inscrite en 1769 dans cette section - où elle porte le $\mathrm{n}^{\circ} 4$ - de son Extrait des ouvres de M. Gellert par Toussaint ${ }^{8}$, l'auteur bien connu des Mours, qui a fait choix de présenter ses traductions (en prose) selon un classement distinguant, au sein des Fabeln de Gellert, apologues, fables, histoires, contes et allégories`. Les divisions retenues par Toussaint sont du reste sujettes à caution : un autre sujet, Le Jeune Homme et le Vieillard, lui aussi traité par Florian ${ }^{\mathrm{IO}}$, se retrouve par exemple dans la section des contes - sous le $\mathrm{n}^{\circ} 34-$ sans qu'il y ait de raison évidente : ce sermon d'un aîné à un homme plus jeune qui s'enquiert des moyens de réussir est bien une fable (ou une parabole) épigrammatique.

L'examen superficiel du second recueil ${ }^{\text {II }}$ du père Barbe, pourtant professeur de rhétorique ${ }^{12}$, ne contribue pas vraiment à nous sortir de notre perplexité grandissante : imitateur avoué des Allemands (Lichtwer, Hagedorn,

6. On trouvera la traduction versifiée de l'original allemand dans le volume de $\mathrm{M}^{\mathrm{me}}$ MercierStevens (Fables et contes de Gellert, traduits en vers par une femme aveugle, Breslau et Leipzig, C. F. Goutsch, I777), p. 62-66.

7. Sous le titre L'Aveugle et le Paralytique (I, 20). Voir J.-N. Pascal, «L'aveugle des fables : un emblème de la nécessaire solidarité entre les hommes", Voir barré, n ${ }^{\circ}$ I8, 1999. $\mathrm{M}^{\mathrm{me}}$ Mercier-Stevens choisit pour titre de sa version en vers L'Aveugle et l'Impotent (ouvr. cité, p. 76-77).

8. Extrait des ceuvres de M. Gellert, contenant ses Apologues, ses Fables et ses Histoires, trad. de l'allemand en français par M. Toussaint, Zullichow, aux dépens de la Maison des Orphelins et de Frommann, 1768 : voir p. 80-8I.

9. Ainsi, chez lui, le conte — d'après Rivery — d'Incle et Yarico porte l'étiquette d' "histoire" (ouvr. cité, III, I, p. I33-I39).

IO. Sous le même titre que le traducteur en prose (I, I7), mais avec un effort sensible de concentration. Voir mon édition récente des Fables de Florian (Ferney-Voltaire, CIEDS, 2005) : pour cette fable comme pour l'essentiel de celles qui sont d'origine étrangère, le texte-source est reproduit après l'apologue français.

II. Fables et contes philosophiques, par M. Barbe, Paris, Delalain, I77I, 216 p. Le poète avait donné auparavant un autre recueil, simplement intitulé Fables nouvelles, en 1762 (Paris, Brocas et Humblot).

I2. On lui doit même un Manuel des rhétoriciens ou Rhétorique moderne (Vitry, Seneuze et Paris, Buttard, 1759-1762). 
Gellert), de l'Anglais Gay et du fabuliste néo-latin Desbillons, c'est dans le sixième et dernier livre de son ouvrage que le poète, qui jusque-là avait utilisé constamment l'étiquette de fable pour se référer à ses textes, utilise celles d'histoire et de conte, dans certaines des notes où il indique ses sources. Cependant, les spécificités typologiques des pièces ainsi désignées ne sautent pas toujours aux yeux... La Soupe au caillou (VI, 3, p. 163-165) comporte une moralité parfaitement conventionnelle, après une narration à caractère d'anecdote qui met en scène un paysan auquel deux étrangers apprennent à faire une bonne soupe bien assaisonnée malgré l'absence de viande, qu'on remplace par un gros caillou :

Dans mille occasions, pour se tirer d'affaire,

Un peu d'esprit est nécessaire.

C'est le cas de la plupart des textes de cet ensemble, avec lesquels contrastent seulement deux morceaux relativement longs - et tous deux entièrement en alexandrins —, intitulés La Patience mise à l'épreuve (VI, p. I68-173) et La Politique criminelle (VI, 6, p. I73-I77), qui sont en effet des contes orientaux, dépourvus de moralité explicite, empruntés à des périodiques britanniques, mais que le poète ne juge bon d'étiqueter dans aucun des deux cas!

Chez Le Monnier ${ }^{13}$, dont le surprenant recueil en prend souvent à son aise avec les pratiques génériques habituelles — il multiplie les pièces longues et les récits qui miment, non sans réalisme, des anecdotes vécues seuls trois textes sont placés par l'auteur sous la rubrique contes, entre quarante-quatre fables et six épîtres badines... Le liminaire, intitulé De la fable, établit quant à lui, sans jamais prononcer le mot conte, que la fable est un non-genre ${ }^{14}$ : elle n'a, pour cet ami de Diderot, ni définition ni poétique et le fabuliste peut raconter ce qu'il veut comme il le veut, pourvu qu'elle illustre une morale "pure et saine» (p. Xvi). Et nous voilà ramenés à la formule de La Fontaine à laquelle je faisais allusion en commençant : une narration libre qui doit conduire à un précepte. Le Monnier - comme beaucoup de ses contemporains : l'âge des poétiques de la fable, chez les

13. Fables, contes et épîtres, par M. l'abbé Le Monnier, Paris, Jombert père, Cellot et Jobert fils aîné, I773, XXIV-2I5 pages (édition in- $8^{\circ}$ ordinaire : il y a eu la même année une édition de prestige, typographiquement distincte, avec un frontispice gravé).

I4. Dans un article où il recense sans ménagement l'Almanach des Muses pour i780, le rédacteur de l'Année littéraire (I780, vol. I, p. 59) s'acharne contre le malheureux Sélis, professeur métromane de l'époque, qui a commis une fable maladroite publiée par le périodique (les italiques sont dans le texte) : «Fable : c'est le nom trivial qu'on donne à présent à toutes les rimailles hermaphrodites, qui n’appartiennent décidément à aucun genre.» 
fabulistes (La Motte, Richer, Rome d'Ardène), ne s'éternise guère au-delà des années 1750 - se débarrasse sans aucun ménagement des règles et des modèles, des typologies et des réflexions génériques. Autant font (ou feront) Dorat ${ }^{15}$ - conteur patenté en vers comme en prose - et Florian - auteur de quelques contes en vers et d'une douzaine de nouvelles pour n'en citer que deux dont les recueils de fables ne comportent pas de mention du conte mais dont les liminaires, à vingt ans de distance (I772 et I792), revendiquent, pour toute poétique, le droit d'admirer La Fontaine et surtout celui de narrer librement ${ }^{16}$.

L'abbé Jean-Louis Aubert (I73I-I8I4), qui commence sa collecte de fables en 1756 pour l'achever - ou presque : il reprendra sporadiquement la plume après la Révolution - en 1773 , détonne un peu dans cet ensemble de fabulistes visiblement peu soucieux de transformer leurs liminaires en cours de rhétorique : dès son recueil inaugural ${ }^{17}$, il ajoute à un rapide Avant-propos (p. 3-I4), de tonalité vindicative et moderne dans lequel il clame son droit à tenter sa chance comme fabuliste après La Fontaine, un long Discours touchant la manière de lire les fables, ou de les réciter (p. 15-43), qui prône une lecture prosaïque, exempte de toute emphase déclamatoire, des apologues versifiés. Implicitement, cet effacement des cadres métriques que souhaite Aubert aboutit à leur remplacement par un soulignement des articulations narratives et des tonalités diverses qui constituent la dramatisation de la fable ${ }^{18}$. Et même si le mot conte n'est jamais prononcé, le Discours laisse deviner — en opposition

15. On remarquera que Dorat et ses imprimeurs ont hésité sur le titre à donner aux quatre livres de Fables du poète: Fables nouvelles, selon une désignation fort répandue au temps des Lumières (qui signifie seulement qu'il s'agit de pièces différentes de celles de La Fontaine, d'adjonctions à la collection inaugurée par le grand fabuliste classique), ou Fables ou Allégories philosophiques (ce qui renvoie d'une part à la procédure rhétorique constitutive du genre et, ironiquement, à une prise de position hostile à la philosophie dominante à laquelle on oppose la "véritable philosophie» des fables). Les exemplaires des Fables de Dorat comportent en général la seconde formule en page de titre et la première en titre courant, mais il en est qui ignorent totalement la seconde, et même d'autres qui se contentent de la simple désignation Fables de M. Dorat au titre... Par ailleurs, la répartition en livres et la numérotation des pièces est variable d'une composition à l'autre : il y aurait là matière à une passionnante enquête bibliologique. Dans certains tirages, on trouve au livre IV, entre les fables 9 et Io, un texte intitulé Le Chemin perdu et retrouvé, étiqueté comme conte. I6. Rappelons seulement la formule de Florian : "Ce genre d'ouvrages est peut-être le seul où les poétiques sont à peu près inutiles.» (Fables, éd. citée, p. 85.)

17. Fables nouvelles, avec un Discours sur la manière de lire les Fables ou de les réciter, par M. ***, Amsterdam et se trouve à Paris, chez Duchesne, 1756, I30 p.

18. Le point faible le plus apparent du Discours d'Aubert — plutôt par mauvaise foi démonstrative que par aveuglement ignorant - est qu'il ne tient guère compte des concordances qu'un bon poète tel que La Fontaine sait établir entre structures syntaxiques ou narratives et structures métriques... 
avec les positions théoriques d'un La Motte, qui n'envisage la construction de la narration que comme une préparation méthodique de l'énoncé de la leçon - un glissement de la fable vers une conception moins uniquement finalisée par la moralité : une sorte d'autonomisation du conte par rapport au précepte, si l'on veut.

Dans les éditions suivantes du recueil d'Aubert, ce liminaire — désormais imprimé après les fables - passe du statut d'ouverture provocatrice à celui, plus discret, d'appendice, mais sans jamais disparaître. L'Avantpropos, quant à lui, prend de l'ampleur dès $176 \mathrm{I}^{\mathrm{I}}$, avec l'adjonction de développements vantant, notamment, les qualités des prédécesseurs $\mathrm{d}^{\prime}$ Aubert ${ }^{20}$, et particulièrement de La Fontaine — dont l'art du récit pour édulcorer le caractère vindicatif de la première mouture. Dans la même édition, divisée en deux parties de trois livres chacune, apparaît à la fin de la première un long texte étiqueté conte moral - l'étiquette précède le titre - et intitulé Thélamon et Zirphé, Hilas et Zénéide (p. I22I30) : histoire édifiante, émaillée de rares incidents, d'un couple pieux et de ses deux enfants qui par leur piété parviennent à obtenir des dieux la guérison de leur pauvre papa victime d'une "fièvre fatale», que le poète dédie à sa vertueuse mère au nom de l'amour qu'il lui porte. Dans l'édition de $1773^{21}$, chacun des trois premiers livres de chacune des deux parties — qui en compte quatre - est désormais terminé par un "conte moral» (l'étiquette placée maintenant après le titre) : Télamon (sic) et Zirphé, Hilas et Zénéide clôture le livre I (p. 39-47); L'Amour paternel ferme le livre II (p. 88-90); L'Accordée de village ${ }^{22}$ termine le livre III (p. I28-I30);

19. Fables nouvelles, divisées en six livres, avec des notes et un Discours sur la manière de lire les Fables ou de les réciter, nouv. éd., revue, corrigée et considérablement augmentée par M. l'abbé Aubert, Paris, Desaint \& Saillant, Duchesne, Langlois Fils, 176I, XXXIV-297 p.

20. C'est à propos de Phèdre qu'Aubert aborde (p. xxirI) «l'art de conter» : «Toutes les fois qu'il s'agit de conter, fussent les choses les plus simples, il faut conter avec grâce, il faut peindre.»

2I. Fables nouvelles, par M. l'abbé Aubert, divisées en huit livres, accompagnées de notes et suivies du Discours sur la manière de lire les Fables ou de les réciter, 4 e éd., considérablement augmentée, Paris, Moutard, I773, XXII-426 p. On remarquera comment l'auteur, anonyme en 1756, placé à la suite du titre en 176I, est au bout du chemin intégré à celui-ci... On notera aussi comment le Discours, en juillet 1773 (date précise de l'impression du volume), est le (fameux) Discours, alors qu'en 1756 et I76I, il n'était qu'un Discours : le journaliste vient d'être désigné pour occuper la chaire de littérature française au Collège royal, où il prononcera son Discours inaugural en décembre (en langue française, alors que ses prédécesseurs étaient restés fidèles au latin).

22. "L'idée de ce conte est prise du tableau de M. Greuze, exposé au Salon du Louvre en I76I, et gravé en I770, par J. J. Flipart", précise Aubert en note (p. I28). La rédaction du texte est probablement postérieure à la gravure. On signalera que l'Avant-propos des Fables nouvelles, dans ses différentes versions, témoigne de l'intérêt de l'abbé Aubert — fils d'un musicien alors fameux — pour la peinture. 
Atys et Damon clôture le livre V (p. 212-216); L'Amour filial ${ }^{23}$ ferme le livre VI (p. 257-260); Colin et Lisette termine le livre VII (p. 297-300). Parallèlement, les livres IV et VIII, ouverts sur des prologues, se concluent sur de brefs épilogues.

Il y a donc un évident effort de structuration "géométrique», qui se double - à considérer les contes — d'un effet de réitération thématique et tonale : les sujets sont toujours les vertus (domestiques, familiales, conjugales), chantées avec une sensibilité molle et édifiante, sans les ambiguïtés et sans l'énergie exaltée qui sauvent parfois de la médiocrité ennuyeuse les textes contemporains d'étiquettes identiques. Or, si l'on observe d'un peu près les ajouts successifs effectués par Aubert à son recueil, on constate qu'ils suivent, justement, une inflexion majoritaire vers le moralisme sensible: la fable dérive progressivement vers le conte moral... Alors qu'à l'origine le poète semblait plutôt tenté par les thématiques religieuses, politiques et sociales — on avait même parfois l'impression qu'il versifiait Montesquieu —, il se met, dès I76I, à privilégier les sujets touchants, souvent restreints à un univers domestique : du Greuze mis en apologue! Le fait, donc, que les Fables nouvelles dans leur version de 1773 soient ponctuées à intervalles réguliers par l'insertion de contes moraux, est évidemment une manière de souligner l'évolution globale du recueil.

Cependant, Aubert, dans l'édition prestigieuse de ses Fables et auvres diverses $^{24}$ imprimée en 1774 pour solenniser son entrée au Collège royal, renonce à cette structuration, pourtant si emblématique de sa démarche : les six contes moraux, ôtés de leur position à la fin des différents livres de fables, se trouvent regroupés au tome II, à une place bien plus conforme à la hiérarchisation traditionnelle qui préside à la constitution des recueils d'«œuvres diverses». Tout se passe comme si le bon abbé avait choisi, une fois atteint le sommet des honneurs, de rentrer dans le rang: les Fables au premier volume (son principal titre de gloire), Psyché (mise en vers du curieux texte hybride de La Fontaine) en tête du second volume, puis $L a$ Mort d'Abel (drame «imité du poème de M. Gessner»), Le Vou de Jephté (sorte de cantate sans doute destinée au Concert spirituel), enfin une section de Poésies diverses (dans cet ordre: Épîtres, Contes moraux, Pièces fugitives), avant le texte du Discours sur les progrès de la langue et de la

23. «Lidée de ce conte est prise du tableau de M. Greuze, exposé au Salon du Louvre en I763, et gravé en I769, par J. J. Flipart", précise Aubert en note (p. 257).

24. Fables et æuvres diverses de M. l'abbé Aubert, Lecteur et Professeur royal en Littérature française, nouv. éd., contenant, entre autres, le poème de Psyché avec des augmentations considérables, et le Discours de l'auteur pour l'ouverture de ses leçons au Collège royal, Paris, Moutard, I774, 2 vol., XXII-340 p. et 340 p., frontispices de Cochin. 
littérature française, qui conclut le recueil et inaugure la nouvelle carrière du poète-journaliste. Le conte, donc, subit une nouvelle éclipse : au lieu d'éclairer de sa lumière problématique le volume des fables, il s'efface dans le bric-à-brac des babioles poétiques qu'on rejette après les parties ostensibles de la production, comme pour faire bonne mesure... Dommage, en tout cas, que l'un des rares exemples, à notre connaissance, de faire jouer le conte - même médiocre - et la fable de manière qui sorte un peu de la topique implicite et floue héritée de la formule de La Fontaine, soit anéantie à peine née.

On avait prévenu : le couple contelfable, vu du côté des fabulistes en vers des Lumières, n'est guère problématique. Il est à craindre qu'il ne le soit pas plus chez les auteurs de poétiques, scolaires ou plus ambitieuses, qui se mettent à pulluler aux alentours de 1750 . On n'a la place, pour conclure ces remarques rapides, que de quelques rappels. Charles Batteux, dans son très didactique Cours de Belles-Lettres (I748), professe que la fable est un récit allégorique tout entier tendu vers la moralité. Et de traiter, par le précepte puis par l'exemple — il analyse plusieurs fables de Phèdre, La Fontaine et La Motte - , des "qualités essentielles ${ }^{25}$ » que doit avoir un récit, pour en conclure que La Fontaine est l'incontestable maître du genre. Jean-François Marmontel, dans sa Poétique française (I763), moins ouvertement pédagogique, où le bref chapitre sur la fable renvoie pour l'essentiel au Discours de La Motte, définit seulement l'apologue comme "un petit poème, où avec l'air d'une simplicité crédule, on présente une vérité morale sous le voile d'un conte ingénu ${ }^{26}{ }^{2}$. L'abbé Jean-Antoine de La Serre (I722-I782), auteur d'une Poétique élémentaire (I77I) d'esprit plutôt moderne — notamment par l'ouverture aux littératures étrangères place sans hésiter son développement consacré aux «avantages de l'apo$\operatorname{logue}^{27}$ » sous la formule fameuse de La Fontaine (VI, I), qu'il cite du reste inexactement :

Une morale nue apporte de l'ennui :

Le conte fait passer la morale $[s i c]$ avec lui.

25. Cours de Belles-Lettres ou Principes de la Littérature, nouv. éd., Paris, Desaint \& Saillant, et Durand, I753, t. I, p. 218.

26. Poétique française, par M. Marmontel, Paris, Lesclapart, I767, t. II, p. 327. On soulignera l'intérêt de cette définition d'apparence simpliste, qui relie clairement le conte au paradigme de l'allégorie.

27. Poétique élémentaire, par M. L* S**, de plusieurs académies, Lyon, les Frères Périsse, p. I37. 
Et l'on perçoit très clairement que, dans un ouvrage consacré pourtant aux règles des genres, il est fortement tenté de prêcher la liberté, par exemple s'agissant de l'épineuse question de la longueur de l'apologue :

Le goût ne s'assujettit point à des règles rigoureuses. On n'allonge point un récit inutilement, lorsque les ornements qu'on lui prête tournent au profit des vérités qu’on développe, ou des vertus que l'on veut inspirer. Une route agréable n'est jamais longue ${ }^{28}$.

L'auteur anonyme - et qui a bien fait de le rester : il ne fait souvent que remanier et résumer sans adresse particulière l'ouvrage de La Serre ${ }^{29}$ en y ajoutant une rigueur didactique héritée de Batteux - de la Poétique élémentaire à l'usage des collèges (178I) reprend (inexactitude comprise!) la citation de La Fontaine en la déplaçant et insiste sur les «qualités et ornements du récit ${ }^{30}$ ".

Bref, les professeurs ne prêtent visiblement guère plus d'attention à notre couple fantomatique que les fabulistes de leur temps, même s'ils consacrent tous un chapitre à la fable... Ils persistent, comme les poètes, à considérer implicitement, malgré tous leurs efforts pour réglementer l'art de la «narration dans le genre familier ${ }^{3 \mathrm{I}}$ » et pour percer le secret du naif et de la gaité, que ce non-genre — que Boileau avait négligé dans son Art poétique - ne se laisse vraiment réduire à aucune définition. Comme le conte, justement, et c'est sûrement le secret de leur succès pérenne à tous deux.

28. Ouvr. cité, p. I47-I48.

29. Il y a peu d'apparence que La Serre ait eu part lui-même à ce démarquage de son ouvrage de I77I.

30. Poétique élémentaire à l'usage des collèges, Metz, J.-B. Collignon, I78I, p. 96.

31. C'est le titre du chapitre sur la fable des Ornements de la mémoire ou les Traits brillants des poètes français les plus célèbres, avec des dissertations sur chaque genre de style, ancêtre des manuels anthologiques de littérature publié pour la première fois (par P.-A. Alletz) en 1749, et réimprimé un nombre incalculable de fois jusqu'à fort tard dans le $\mathrm{XIX}^{\mathrm{e}}$ siècle. 